\title{
The Drawing Behavior of Polyvinylalcohol Fibers
}

\author{
R. SCHELLEKENS * and C. BASIIAANSEN \\ DSM Research, P.O. Box 18, 6160 MD, Geleen, The Netherlands
}

\begin{abstract}
SYNOPSIS
The solid-state drawing behavior and properties of solution-spun polyvinylalcohol fibers were investigated. A comparison was made with solution-spun, ultra-drawn polyethylene fibers. The maximum attainable draw ratio of polyvinylalcohol fibers is low $(\sim 20)$, even at optimized conditions with respect to polymer concentration in solution. In contrast to polyethylene, the maximum attainable draw ratio hardly increases with increasing molecular weight. However, high modulus $(\sim 70 \mathrm{GPa})$ and strength $(\sim 2.3 \mathrm{GPa})$ polyvinylalcohol fibers can be produced, despite the low maximum attainable draw ratio. It is suggested that the observed phenomena, with respect to both the drawing behavior and properties of polyvinylalcohol fibers, originate from intermolecular hydrogen bonds in the polymer.
\end{abstract}

\section{INTRODUCTION}

The solid-state drawing behavior of semicrystalline, flexible polymers has been investigated extensively in the past. ${ }^{1-10}$ The main objective of these studies was to produce structures with a high level of chain orientation and extension in order to approach the theoretical modulus and strength of polymers.

Most studies on the drawing behavior of semicrystalline polymers focused on linear polyethylenes. Experimental procedures to produce structures with Young's moduli and tensile strengths which approach the theoretical values have been conducted. ${ }^{1-10,13-16}$ For instance, with the so-called "gelspinning process," polyethylene structures can be produced with a Young's modulus and tensile strength of $100-150 \mathrm{GPa}$ and $4-5 \mathrm{GPa},{ }^{6-10}$ respectively.

The drawing behavior of polar polymers, such as polyamides, polyacrylonitrile, polyethylene terepthlate, and polyvinylalcohol, has received relatively little attention in the past. As does polyethylene, these materials possess a high theoretical modulus in the chain direction. ${ }^{11,17,18}$ Moreover, high modulus and strength fibers produced from these materials might offer some advantages over polyethylene fi-

* To whom correspondence should be addressed.

Journal of Applied Polymer Science, Vol. 43, 2311-2315 (1991) (c) 1991 John Wiley \& Sons, Inc. CCC $0021-8995 / 91 / 122311-05 \$ 04.00$ bers, such as a higher melting point and less creep. Unfortunately, most attempts to produce strong and stiff fibers from these materials were rather unsuccessful. In most cases the maximum attainable Young's modulus $(<20 \mathrm{GPa})$ and tensile strength $(<1 \mathrm{GPa})$ were rather low. ${ }^{19-22}$

Recently, it was shown that solution-spun, drawn polyvinylalcohol fibers can be produced possessing Young's moduli and tensile strengths up to $70 \mathrm{GPa}$ and $2.3 \mathrm{GPa}$, respectively. ${ }^{23-27}$ In the present study, the solid-state drawing behavior of polyvinylalcohol fibers is investigated. A comparison is made between the properties of drawn polyethylene and polyvinylalcohol fibers.

\section{EXPERIMENTAL}

Several polyethylene and polyvinylalcohol grades were used in the spinning experiments. These polymers were synthesized at DSM Research. Some relevant characteristics of the polymers used in this study are listed in Table I.

Polyethylene solutions were prepared by dissolving the material in decalin at $160^{\circ} \mathrm{C}$. Prior to the dissolution procedure, an antioxidant (di-butyl- $p$ cresol) was added to the polymer and the polymersolvent mixtures were degassed at room temperature. After complete dissolution occurred, which took approximately two hours, the solutions were transferred to a fiber extrusion device and spun at 
Table I Polyethylene and Polyvinylalcohol Grades Used in this Study

\begin{tabular}{rrrr}
\hline Grade & $\begin{array}{c}\mathrm{DP}_{w} \\
{[-]}\end{array}$ & $\begin{array}{c}M_{w} \\
{[\mathrm{~kg} / \mathrm{mol}]}\end{array}$ & $\begin{array}{c}c^{*} \\
{[\% \mathrm{w} / \mathrm{w}]}\end{array}$ \\
\hline PE: a & 3500 & 100 & 20 \\
b & 14,500 & 400 & 8 \\
c & 28,000 & 800 & 4 \\
d & 60,000 & 2000 & 1 \\
PVAL: e & 1300 & 57 & 27 \\
f & 3400 & 149 & 15 \\
g & 13,000 & 572 & 6 \\
h & 29,000 & 1270 & 4 \\
\hline
\end{tabular}

$160^{\circ} \mathrm{C}$. Subsequently, the fibers were quenched to room temperature and dried at ambient conditions.

In the case of polyvinylalcohol, ethylene glycol was used as a solvent and solutions were spun at $160^{\circ} \mathrm{C}$. After spinning, the fibers were quenched/ extracted in methanol and dried at ambient conditions. Special care was taken in these experiments to remove all residual traces of ethylene glycol.

The minimum concentration from which coherent fibers can be spun was determined, by trial and error, for all the polymer grades used in this study. The polymer concentration was minimized in order to obtain optimum conditions for drawing. ${ }^{6-10,28}$ The polymer concentrations used in the spinning experiments are listed in Table I.

Dry polyethylene and polyvinylalcohol fibers were drawn on thermostatistically-controlled, hot shoes at $100^{\circ} \mathrm{C}$ and $190^{\circ} \mathrm{C}$, respectively. The draw ratio was determined by measuring the displacement of ink-marks.

Stress-strain curves of drawn fibers were recorded on a Zwick Tensile Tester, equipped with fiber clamps. Prior to the tensile testing, polyvinylalcohol fibers were conditioned for $24 \mathrm{~h}$ at a relative humidity of $65 \%$. The initial length of the fibers was $0.2 \mathrm{~m}$ and a constant crosshead speed of $0.02 \mathrm{~m} /$ min was used.

\section{RESULTS}

The minimum polymer concentration in solution from which coherent, drawable polyethylene and polyvinylalcohol fibers can be spun $\left(c^{*}\right)$ was determined experimentally in order to obtain optimum conditions for drawing. ${ }^{7-10}$ In Table $I$, it is shown that this optimum polymer concentration in solution decreases with increasing degree of polymerization.

The maximum attainable draw ratio of semi- crystalline polymers increases with increasing drawing temperature. ${ }^{9}$ However, relaxation phenomena start to occur at temperatures close to or above the melting temperature and, consequently, the drawing effectiveness decreases. ${ }^{9}$ In order to obtain optimum drawing conditions, both the polyethylene and polyvinylalcohol fibers are drawn at approximately $30-40^{\circ} \mathrm{C}$ below their respective melting temperatures (see Experimental).

The maximum attainable draw ratio of linear polyethylenes increases rapidly with increasing molecular weight at optimum conditions with respect to polymer concentration in solution ${ }^{28}$ (Fig. 1). However, the maximum attainable draw ratio of polyvinylalcohol fibers is low $(\sim 20)$ and hardly increases with increasing degree of polymerization (Fig. 1).

In Figure 2, stress-strain curves of drawn polyvinylalcohol and polyethylene fibers are shown. The fibers possess an identical degree of polymerization $(\sim 3500)$ and draw ratio $(\sim 20)$. Nevertheless, large differences are observed in the stress-strain curves of the drawn fibers at room temperature. The Young's modulus of drawn polyvinylalcohol and

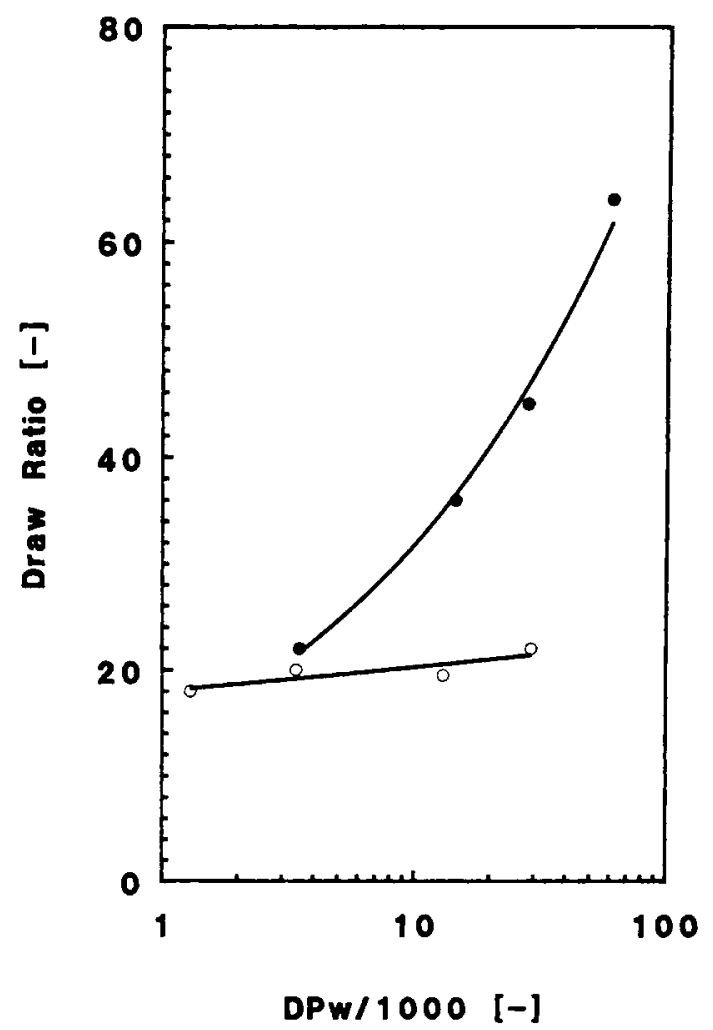

Figure 1 Maximum attainable draw ratio of polyethylene (๑) and polyvinylalcohol (O) fibers $\left(T_{d}=100^{\circ} \mathrm{C}\right.$ and $190^{\circ} \mathrm{C}$, respectively). 


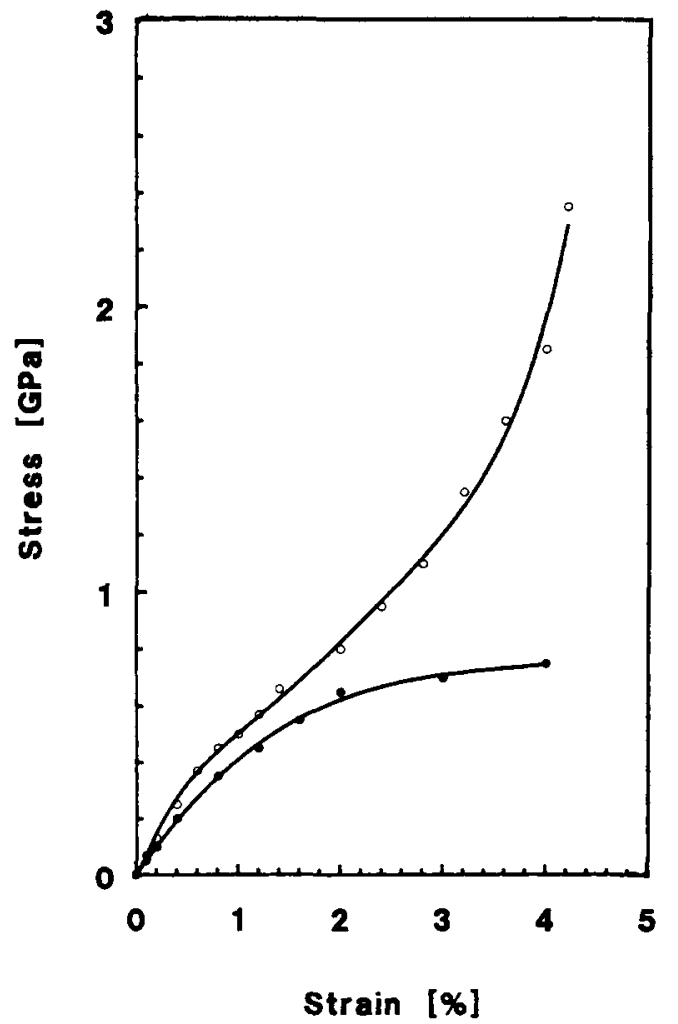

Figure 2 Stress-strain curves of drawn polyethylene (๑) and polyvinylalcohol (O) fibers (draw ratio $=20, \mathrm{DP}_{w}$ $=3500$ ).

polyethylene fibers is plotted in Figure 3 as a function of the draw ratio. Compared with linear polyethylene, the Young's modulus of polyvinylalcohol fibers increases rapidly with increasing draw ratio. Consequently, polyvinylalcohol fibers with a high Young's modulus can be obtained despite the low maximum attainable draw ratio. The tensile strength of polyethylene and polyvinylalcohol fibers, with a degree of polymerization of approximately 3500 , is shown in Figure 4 as a function of draw ratio. This figure indicates that fibers with a high tensile strength can be obtained using a polyvinylalcohol grade with a low molecular weight.

\section{DISCUSSION}

In the case of high molecular weight polyethylenes $\left(M_{w}>5 \times 10^{2} \mathrm{~kg} / \mathrm{mol}\right)$, the maximum attainable draw ratio can be enhanced drastically by spinning from semidilute solutions (Fig. 1) ${ }^{6-10,28}$ Apparently, the constraints that limit the maximum attainable draw ratio of meltcrystallized polyethylenes can be removed by using appropriate dissolution proce-

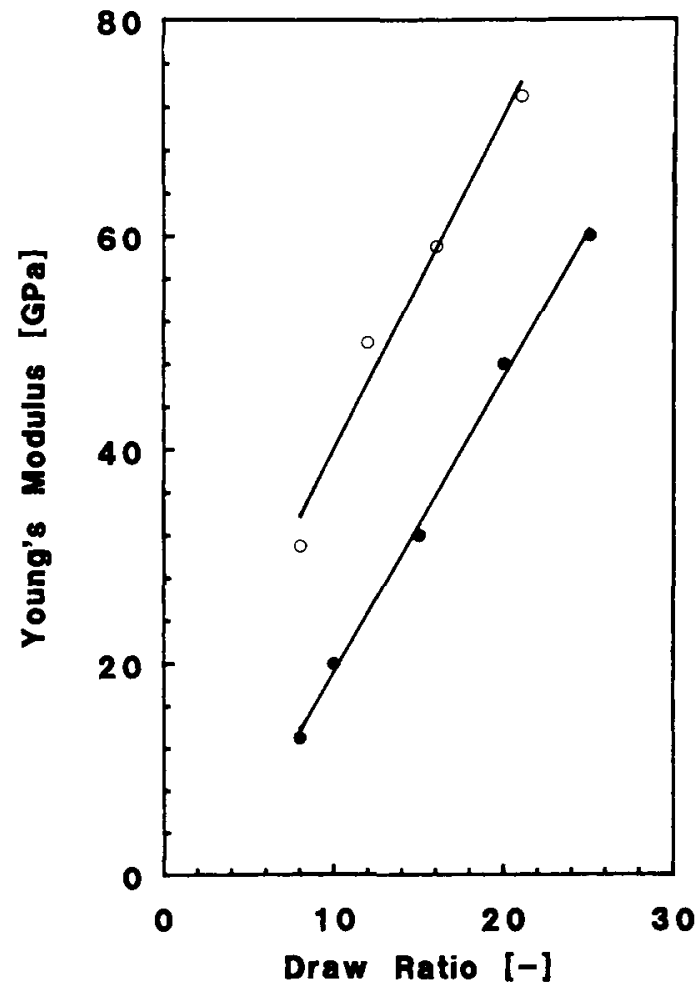

Figure 3 Young's modulus of polyethylene ( $)$ and polyvinylalcohol $(\mathrm{O})$ fibers as a function of the draw ratio.

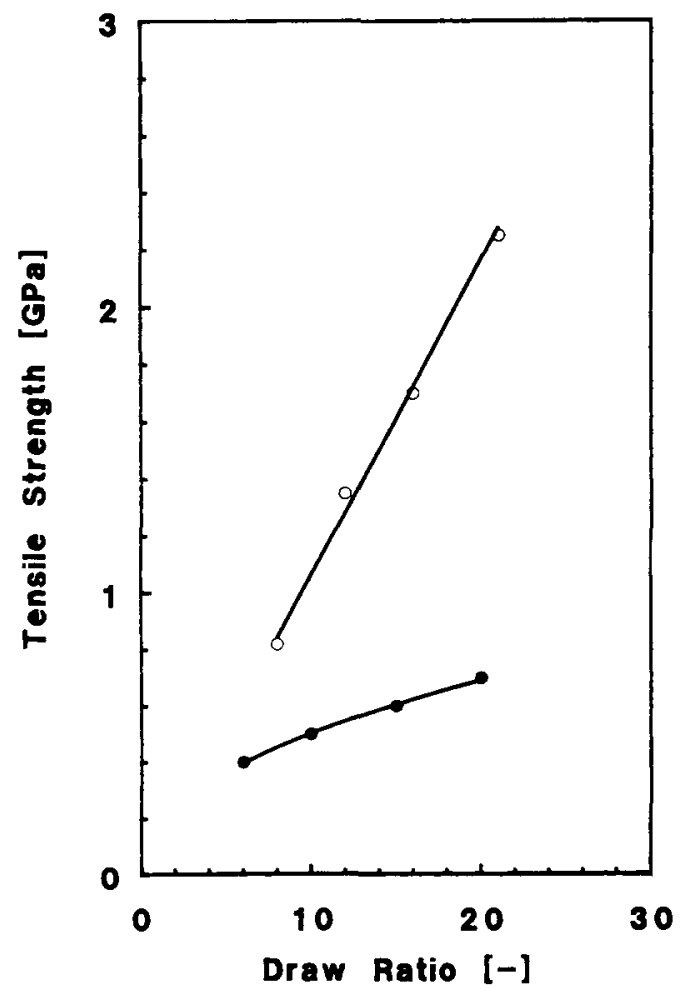

Figure 4 Tensile strength of drawn polyethylene (•) and polyvinylalcohol $(O)$ fibers $\left(\mathrm{DP}_{w}=3500\right)$. 
dures. Moreover, the limited intermolecular interaction within the polyethylene crystal lattice (Van der Waals forces) facilitates chain slippage and unfolding of lamellae during drawing at elevated temperatures. Consequently, fibers with a high degree of chain orientation and extension and with excellent mechanical properties can be obtained.

In the polyvinylalcohol crystal lattice, hydrogen bonds are present, which resist shear deformation and intermolecular chain slippage. Consequently, the unfolding of lamellae during solid state drawing is restricted, which results in a low, and molecular weight independent, maximum attainable draw ratio (Fig. 1). However, the limited maximum attainable draw ratio of polyvinylalcohol fibers does not result in poor mechanical properties after drawing. In Figures 3 and 4 it is shown that the maximum attainable Young's modulus ( $\sim 70 \mathrm{GPa})$ and tensile strength $(\sim 2.3 \mathrm{GPa})$ of polyvinylalcohol fibers are high, despite the low maximum attainable draw ratio. A substantial increase in tensile strength and Young's modulus is observed in comparison with polyethylene fibers with an identical draw ratio and degree of polymerization $\left(\mathrm{DP}_{w}=3500\right)$.

Recently, a model was proposed by Smith et al. to describe the Young's modulus vs. draw ratio curves of flexible polymers. ${ }^{29}$ In this model it is assumed that drawn fibers consist of a series connection of high ("helix") and low modulus ("coil") elements. The "helix" phase consists of perfectly oriented crystals. Misaligned crystals and (oriented) amorphous material are represented in this model by the "coil" elements. In the case of polyethylene and polyvinylalcohol, the modulus of the stiff "helix" phase is virtually identical. ${ }^{17,18}$ Apparently, the "coil" modulus of polyvinylalcohol is high in comparison with polyethylene. This increased "coil" modulus probably originates from intermolecular hydrogen bonds, which, for instance, enhance the shear modulus of the misaligned crystals. ${ }^{17}$

The degree of intermolecular interaction in drawn fibers also influences their large strain properties and fracture behavior (Figs. 2 and 4 ). In the stressstrain curve of polyethylene fibers a yield point is observed (Fig. 2). Such a yield point is usually correlated with shear deformation and excessive intermolecular chain slippage within the crystal lattice. ${ }^{12}$ In the stress-strain curve of drawn polyvinylalcohol fibers an inflection point is observed at a strain of approximately $0.5 \%$. After the inflection point, the stress continues to increase with increasing strain. Apparently, at large strains ( $>1 \%)$, the hydrogen bonds prevent excessive intermolecular chain slippage in the crystal lattice. A pronounced yield point, comparable with polyethylene, is not observed and the fibers fracture in a brittle fashion. The high tensile strength of polyvinylalcohol fibers, in comparison with polyethylene, probably originates from a transition in fracture mechanism, from a chain slippage to a chain scission-dominated failure process, which is caused by the increased intermolecular interaction.

A few critical remarks are in order with respect to the experimental data reported in this study. In recent studies, ${ }^{30,31}$ it was shown that polyvinylalcohol fibers with Young's moduli and tensile strengths, which exceed the maximum values quoted in this study, can be produced. Unfortunately, rather exotic processing and/or drawing procedures were used in these studies, which severely limit their application.

\section{CONCLUSIONS}

In contrast to polyethylene, the solid-state drawing behavior of polyvinylalcohol fibers cannot be enhanced drastically by spinning from semidilute solutions. However, despite the low maximum attainable draw ratio, fibers with Young's moduli of up to $70 \mathrm{GPa}$ and tensile strengths of over $2 \mathrm{GPa}$ can be produced. It is suggested that the observed phenomena, with respect to the drawing behavior and properties of polyvinylalcohol fibers, are related to the presence of intermolecular hydrogen bonds in the material.

\section{REFERENCES}

1. G. Capaccio and I. M. Ward, Nature (Phys. Sci.), 243, 143 (1973).

2. G. Capaccio and I. M. Ward, Polym., 15, 233 (1974).

3. G. Capaccio and I. M. Ward, Polym., 16, 239 (1975).

4. G. Capaccio and I. M. Ward, Polym. Eng. Sci., 15, 219 (1975).

5. G. Capaccio, T. A. Crompton, and I. M. Ward, J. Polym. Sci. Phys. Ed., 14, 1641 (1976).

6. DSM/Stamicarbon, US Pat. 4,344,908; 4,422,933; 4,430,383; 4,436,689; Inv. P. Smith and P. J. Lemstra.

7. P. Smith, P. J. Lemstra, and H. C. Booy, J. Polym. Sci. Phys. Ed., 19, 877 (1981).

8. P. Smith, P. J. Lemstra, B. Kalb, and A. Pennings, Polym. Bull., 1, 733 (1979).

9. P. Smith and P. J. Lemstra, Polym., 21, 1341 (1980).

10. P. Smith and P. J. Lemstra, Coll. Polym. Sci., 258, 891 (1980).

11. K. Tashiro and H. Tadokoro, Macrom. 11, 914 (1978).

12. A. J. Kinloch and R. J. Young, Fracture Behavior of Polymers, Applied Science, New York, 1983. 
13. P. D. Griswold, A. E. Zachariades, and R. S. Porter, Polym. Eng. Sci., 18, 861 (1978).

14. A. E. Zachariades, P. D. Griswold, and R. S. Porter, Polym. Eng. Sci., 18, 861 (1978).

15. H. H. Chuah and R. S. Porter, Polym., 27, 1002 (1986).

16. A. Zwijnenburg and A. J. Pennings, Coll. Polym. Sci., 254, 868 (1976).

17. K. Tashiro and H. Tadokoro, Macrom., 19, 481 (1981).

18. K. E. Perepelkin, Ang. Macrom. Chem., 22, 181 (1972).

19. D. C. Prevorsek, Y. D. Kwon, and R. K. Sharma, J. Mater. Sci., 12, 2310 (1977).

20. A. Acierno, F. P. Mantia, G. Polzzotti, and A. Cifferi, J. Polym. Sci. Phys. Ed., 17, 1903 (1979).

21. S. Gogolewski and A. J. Pennings, Polym., 26, 1394 (1985).

22. D. Hofman, U. Goschel, E. Walenta, D. Geiss, and B. Phillips, Polym., 30, 242 (1989).
23. Y. D. Kwon, S. Kavesh, and D. C. Prevorsek, USP $4,440,711$.

24. I. Sakurada, Polyvinylalcohol Fibers, Marcel Dekker, New York, 1985.

25. R. Schellekens, H. J. Rutten, and P. J. Lemstra, DSM Stamicarbon, EP 212,757.

26. F. C. H. Mokveld and R. Schellekens, DSM Stamicarbon, EP 216,407.

27. R. Schellekens, and H. Ketels, Polym. Comm., 31, $212(1990)$.

28. C. W. M. Bastiaansen, J. Polym. Sci. Phys. Ed., to appear.

29. P. A. Irvine and P. Smith, Macrom., 26, 240 (1986).

30. T. Kunugi and T. Kawasumi, IUPAC, 32nd International Symposium on Macromolecules, Kyoto, Japan, 1-6 August 1988.

31. K. Yamaura, T. Tanigami, N. Hayashi, K. I. Kosuda, and S. Matsuzawa, J. Appl. Pol. Sci., 40, 905 (1990).

Accepted February 7, 1991 